- Original Article

\title{
Validation of the Simplified Palliative Prognostic Index to Predict Survival for Advanced Cancer Patients in Home Hospice Setting
}

\author{
Hyeon-Jeong Yang, Seok-Joon Yoon*, Jong-Sung Kim, Sung-Soo Kim, Jin-Gyu Jung, Won Yoon Suh, Sami Lee, \\ Hyun Gu Kim, Yong Woo Lee \\ Department of Family Medicine, Research Institute for Medical Science, Chungnam National University School of Medicine, Daejeon, Korea
}

\begin{abstract}
Background: The simplified Palliative Prognostic Index (sPPI) substitutes a single item from the Communication Capacity Scale (CCS) for the delirium item of the original PPI. This study aimed to examine the validity of the sPPI for patients with advanced cancer in a home-based hospice care setting.

Methods: This study included 75 patients with advanced cancer who received home-based hospice care. We used medical records maintained by professional hospice nurses who had visited the patients in their homes. Based on their sPPI score, patients were divided into three groups-A $(<4)$, B ( $\geq 4$ and $<6)$, and C $(\geq 6)-$ to compare survival. Further, we investigated the sPPI's accuracy using the area under the receiver operating characteristic curve (AUC) and sensitivity and specificity for 3- and 6-week survival. We used three sPPIs including different substitutions for the delirium item (two methods using the CCS and one using the Korean Nursing Delirium Screening Scale).

Results: The median survival was 60-61 days for group A, 27-30 days for group B, and 12-16 days for group C. The difference in survival was significant $(\mathrm{P}<0.05)$. The AUC was $0.814-0.867$ for 3 -week survival and $0.736-0.779$ for 6-week survival. For 3- and 6-week survival, prognostic prediction showed sensitivities of 76.2\%-90.9\% and 76.3\%$86.8 \%$, and specificities of $64.2 \%-88.7 \%$ and $51.4 \%-70.3 \%$, respectively.

Conclusion: The sPPI, which is measured by professional hospice nurses, has acceptable validity to predict survival for patients with advanced cancer in a home hospice setting in South Korea.
\end{abstract}

Keywords: Palliative Care; Hospice Care; Prognosis; Survival

Received: July 13, 2020, Revised: August 11, 2020, Accepted: September 1, 2020

*Corresponding Author: Seok-Joon Yoon https://orcid.org/0000-0002-6784-8191

Tel: +82-42-280-8371, Fax: +82-42-280-7879, E-mail: sj0219@cnuh.co.kr 


\section{INTRODUCTION}

In palliative care, the accurate prediction of life expectancy is important for patients, families, and healthcare providers. If life expectancy can be estimated more precisely, patients can plan the remaining years or months of their lives and prepare for their death. ${ }^{1)}$ Moreover, families can prepare to bid farewell to their loved ones and handle practical concerns such as the caregiving period and medical expenses. ${ }^{2)}$ In addition, it is important for healthcare providers to accurately predict life expectancy to determine treatment plans and provide appropriate medical care. ${ }^{3)}$

Accordingly, various tools for predicting the prognosis of patients with terminal cancer have been studied and developed. The Palliative Prognostic Index (PPI), ${ }^{4)}$ Cancer Prognostic Scale, ${ }^{4)}$ Palliative Performance Scale (PPS), ${ }^{5)}$ Palliative Prognostic (PaP) Score, ${ }^{6)}$ PaP Score with Delirium (D-PaP), Japan Palliative Oncology Study-Prognostic Index ${ }^{8)}$ and Prognosis in Palliative Care Study (PiPS ${ }^{9)}$ model are some that have proven effective. Among them, the PPI is used worldwide for its simplicity and high predictive power. ${ }^{10-14)}$ The PPI was proposed by Morita et al. ${ }^{15)}$ of Japan and uses five variables: the PPS, oral intake, edema, dyspnea at rest, and delirium. The PPI does not include blood or imaging tests, and is thus applicable to patients in various conditions.

The diagnosis of delirium, which is one of the variables of the PPI, is based on the Diagnostic and Statistical Manual of Mental Disorders, fourth edition (DSM-IV). According to the definition in the DSM-IV, patients are diagnosed with delirium if there is a change in consciousness and cognitive function, along with reduced attention and concentration. These symptoms appear within hours or days. ${ }^{16)}$ However, since the DSM is a diagnostic tool, it may not be suitable for quick assessment at the point of treatment, and it may be difficult for certain healthcare providers to make judgments based on the diagnosis.

Morita et al. ${ }^{17)}$ demonstrated the utility of the Communication Capacity Scale (CCS) in measuring the ability to communicate in patients with delirium. Hamano et al. ${ }^{18)}$ demonstrated the effectiveness of the simplified PPI (SPPI), which replaces items related to delirium with the CCS. Comparing the sPPI and PPI, Hamano et al. ${ }^{18)}$ discovered that the sPPI's sensitivity and specificity of survival prediction within 3 weeks was $72.9 \%$ and $67.6 \%$, respectively, while these values were $80.3 \%$ and $61.8 \%$, respectively, at 6 weeks. In contrast, the PPI's sensitivity and specificity of survival prediction within 3 weeks was $66.2 \%$ and $76.6 \%$, respectively, and within 6 weeks was $74.2 \%$ and $72.8 \%$, respectively. However, studies on the sPPI have been insufficient, and further research is needed to establish its validity in various environments.

Since the SPPI is easier to evaluate than the PPI, it may be more useful for patients in home-based hospice care, who are in an environment where access to diagnostic equipment or personnel may be limited. However, most previous studies have targeted inpatients receiving hospice care. While Hamano et al. ${ }^{19)}$ analyzed the utility of the PPI for patients in home-based hospice care, there are no such studies on the sPPI.
Accordingly, the purpose of this study was to evaluate the validity of the sPPI's life expectancy prediction for patients in home-based hospice care at a university hospital in South Korea.

\section{METHODS}

\section{Subjects}

The subjects of this study were 75 patients who received home-based hospice care from a university hospital from January 2019 to March 2020. The survival period was defined from the day when home-based hospice care began to the day of death. The patients were followed up for 6 months if they had survived at the end of the home-based hospice care period. This study was approved by the institutional review board of Chungnam National University Hospital (IRB approval no., 2018-08-023). Informed consents were collected from patients or their families.

\section{Study Method}

Three professional hospice nurses recorded the sPPI items upon their first home visit, and the additional assessment of delirium was made according to the Korean Nursing Delirium Screening Scale (Korean Nu-DESC). ${ }^{20)}$

The sPPI score was calculated using the PPS, oral intake, edema, dyspnea at rest, and CCS. The PPS was developed by modifying the Karnofsky Performance Scale and covers five aspects: ambulation, activity and evidence of disease, self-care, oral intake, and level of consciousness. ${ }^{21)}$ Based on the patient's overall condition, the score ranges from 0 (death) to 100 (normal); scores between 10 and 20 were given 4 points, scores between 30 and 50 were given 2.5 points, and scores $\geq 60$ were given 0 points. In terms of oral intake, the patient received 2.5 points for severe reduction, 1 point for moderate reduction, and 0 points for normal intake. For edema, 1 point was given for presence and 0 for absence. For dyspnea at rest, 3.5 points were given for presence and 0 for absence. The CCS was divided into four levels: level 1, when explicit and complex communication was possible; level 2, when explicit but simple communication was possible; level 3, when there was a slight incoherency or meaningful communication was possible only with stimulation; and level 4, when speech was out of context or when there was no verbal response even with stimulation. The presence of delirium was determined using the CCS; 4 points were given for presence and 0 point for absence. The total sPPI score ranged from 0 to 15 .

The Nu-DESC is composed of five items: disorientation, inappropriate behavior, inappropriate communication, illusions/hallucinations, and psychomotor retardation. ${ }^{22)} \mathrm{A}$ score between 0 and 2 was assigned according to the degree of each item, but a study that introduced the $\mathrm{Nu}$-DESC in Korea showed that there was no clear standard for determining the severity of each item. ${ }^{20)}$ Hence, it was modified to have a maximum score of 5 points. In this study, the presence of delirium was identified by a score of $\geq 2$ points on the Korean Nu-DESC based on previous findings. In addition, demographic data including age and 
gender, as well as medical history such as primary sites of cancer and treatment methods including surgery, chemotherapy, and radiotherapy, were investigated.

\section{Statistical Analysis}

Based on the study by Hamano et al., ${ }^{18)}$ the patients' sPPI scores were divided into three groups: $<4$ points, $4-6$ points, and $\geq 6$ points. The Kaplan-Meier survival curve was obtained and a log-rank test was performed to compare the prognosis. In addition, the sensitivity, specificity, positive predictive value (PPV), and negative predictive value (NPV) were calculated to verify the accuracy of the sPPI in 3- and 6 -week survival predictions. The receiver operating characteristic (ROC) curve was constructed and the area under the ROC curve (AUC) was measured to verify the discriminative accuracy for 3- and 6-week survival.

There is currently no clear cutoff value for the classification of CCS levels to determine whether delirium is present. When a previous study defined levels 2,3 , and $4(1 / 234)$ or levels 3 and 4 (12/34) as delirium, the accuracy was $87 \%$ and $75 \%$, respectively. ${ }^{18)}$ In both cases, comparing the sPPI and PPI showed similar predictability; thus, using the $1 / 234$ standard with simple clinical judgment was recommended.

However, according to the Korean Nu-DESC, the frequency of delirium detected was $16 \%$, whereas the frequency after dividing the CCS levels into $1 / 234$ and $12 / 34$ was $48.0 \%$ and $4.0 \%$, respectively. The frequencies of delirium obtained from the three methods-CCS $1 / 234$, CCS 12/34, and the Korean Nu-DESC standard-were quite different. Therefore, the analysis was conducted by applying all three. The use of

Table 1. Patients' characteristics

\begin{tabular}{lc}
\hline \multicolumn{1}{c}{ Characteristic } & Value \\
\hline Age (y) & $69.12 \pm 13.36$ \\
Sex & $39(52.0)$ \\
Male & $36(48.0)$ \\
Female & \\
Primary sites & $19(25.3)$ \\
Lung & $5(6.7)$ \\
Stomach & $1(1.3)$ \\
Esophagus & $13(17.3)$ \\
Colon/rectum & $1(1.3)$ \\
Liver & $7(9.3)$ \\
Gall bladder/bile duct & $11(14.7)$ \\
Pancreas & $4(5.3)$ \\
Breast & $7(9.3)$ \\
Cervix/ovary & $1(1.3)$ \\
Lymphoma/leukemia & $2(2.7)$ \\
Head and neck & $4(5.3)$ \\
Others & \\
Anticancer treatment & $33(56.0)$ \\
Surgery & $45(60.0)$ \\
Chemotherapy & $19(25.3)$ \\
Radiotherapy & $18(24.0)$ \\
No treatment & $39 \pm 7.79$ \\
Median survival (d) &
\end{tabular}

Values are presented as mean \pm standard deviation or number (\%). the following three sPPIs including different diagnostic methods of delirium detection, relatively easier methods than using the DSM-IV standard: (1) sPPI1: sPPI using the CCS 1/234 standard for delirium diagnosis; (2) sPPI2: sPPI using the CCS 12/34 standard for delirium diagnosis; (3) sPPI3: sPPI using a score $\geq 2$ points on the Korean $\mathrm{Nu}$ DESC for delirium diagnosis.

The analysis was performed using IBM SPSS ver. 24.0 (IBM Corp., Armonk, NY, USA), and the significance level for all statistical analyses was set at $<0.05$.

\section{RESULTS}

\section{Characteristics of the Study Subjects}

Of the 75 patients, 39 were male $(52.0 \%)$ and the average age was 69.12 years. The most prevalent site of primary cancer was the lung (19 patients, $25.3 \%)$. As for treatments, 33 patients $(44.0 \%)$ had undergone surgery, 45 had received chemotherapy (60.0\%), 19 had received radiotherapy (25.3\%), and $18(24.0 \%)$ had not received any treatment (Table 1). The median survival period was 39 days; 22 patients survived for 3 weeks or less (29.3\%), while 38 patients survived for 6 weeks or less (50.7\%).

The average age of the three professional hospice nurses who participated in the survey was 43.7 years, and the average number of years of clinical experience was 19.7. The average number of years of hospice experience was 10.3, and the average number of patients under their charge per year was 80 .

Table 2. Prevalence of items on the simplified Palliative Prognostic Index

\begin{tabular}{llr}
\hline \multicolumn{1}{c}{ Item } & Score & No. (\%) \\
\hline Palliative Performance Scale & & \\
$10-20$ & 4.0 & $8(10.7)$ \\
$30-50$ & 2.5 & $33(44.0)$ \\
$\geq 60$ & 0 & $34(45.3)$ \\
Oral intake & & \\
$\quad$ Severely reduced & 2.5 & $29(38.7)$ \\
Moderately reduced & 1.0 & $38(50.7)$ \\
Normal & 0 & $8(10.7)$ \\
Edema & & \\
$\quad$ Present & 1.0 & $33(44.0)$ \\
Absent & 0 & $41(54.7)$ \\
Dyspnea at rest & & \\
$\quad$ Present & 3.5 & $20(26.7)$ \\
Absent & 0 & $55(73.3)$ \\
Delirium & & \\
Present & & \\
$\quad$ CCS 234 & 4.0 & $36(4.0)$ \\
CCS 34 & & $12(16.2)$ \\
$\quad$ Korean Nu-DESC & & \\
Absent & &
\end{tabular}

CCS 234 diagnosis of delirium as levels 2, 3, 4 on the CCS; CCS 34 diagnosis of delirium as levels 3 and 4 on the CCS; Nu-DESC diagnosis of delirium as $\geq 2$ points on the Korean Nu-DESC.

CCS, Communication Capacity Scale; Korean Nu-DESC, Korean Nursing Delirium Screening Scale. 


\section{Relationship between sPPI Score and Survival Time}

Table 2 shows the results of the sPPI measurement. The patients were divided into three groups: those with an SPPI score of $<4$ points in group A, those with an sPPI score of 4-6 points in group B, and those with an sPPI score of $\geq 6$ points in group C. Each group was analyzed using the Kaplan-Meier method to obtain the survival curves shown in Figure 1. For sPPI1, the median survival duration was 60 days for group A (95\% confidence interval [CI], 39.8-80.2; $\mathrm{n}=24), 27$ days for group B (95\% CI, 13.4-40.6; $\mathrm{n}=12$ ), and 16 days for group C (95\% CI, 3.8-28.2; $\mathrm{n}=39$ ). For sPPI2, the median survival duration was 61 days for group $\mathrm{A}$ (95\% CI, 49.4-72.6; $\mathrm{n}=35$ ), 30 days for group B (95\% CI, 17.9-42.1; $\mathrm{n}=17)$, and 12 days for group C (95\% CI, 4.17-19.8; $\mathrm{n}=23$ ). For sPPI3, the median survival duration was 60 days for group A (95\% CI, 50.369.7; $\mathrm{n}=32$ ), 27 days for group $\mathrm{B}$ (95\% CI, 11.3-42.7; $\mathrm{n}=16$ ), and 16 days for group C (95\% CI, 7.75-20.3; n=26). The log-rank test showed a significant difference in survival duration for each group for all three sPPIs $(\mathrm{P}<0.05)$.
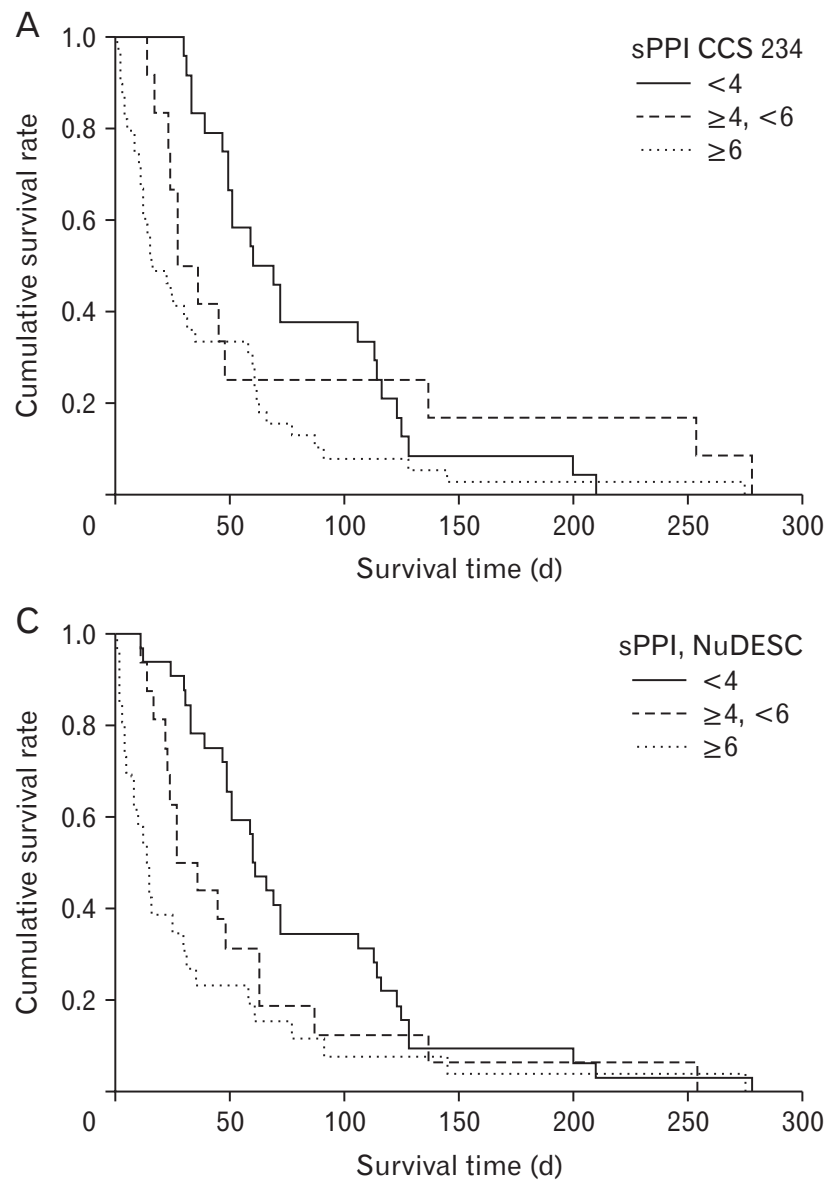

For the prediction of life expectancy at 6 weeks or less when the sPPI score was $\geq 4$, sensitivity was highest in sPPI1 (86.8\%), specificity was highest in sPPI2 (70.3\%), PPV was highest in sPPI2 (72.5\%), and NPV was highest in sPPI1 (79.2\%). For the prediction of life expectancy at 3 weeks or less when the sPPI score was $\geq 6$, sensitivity was highest in sPPI1 (90.9\%), specificity was highest in sPPI2 (88.7\%), PPV was highest in sPPI2 (73.9\%), and NPV was highest in sPPI1 (94.4\%). The overall accuracy of an sPPI2 score of $\geq 4$ for predicting 6 -week survival and of an sPPI2 score $\geq 6$ for predicting 3 -week survival was highest among the three sPPIs: $73.4 \%$ and $85.4 \%$, respectively (Table 3 ).

The AUC of sPPI1 was calculated as 0.736 for 6 -week survival (95\% CI, 0.623-0.849) and 0.861 for 3-week survival (95\% CI, 0.777-0.944). The AUC of sPPI2 was calculated as 0.779 for 6 -week survival (95\% CI, 0.671-0.887) and 0.867 for 3-week survival (95\% CI, 0.777-0.956); these were the highest among the three sPPIs. The AUC of sPPI3 was calculated as 0.758 for 6-week survival (95\% CI, 0.646-0.870) and 0.814 for 3-week survival (95\% CI, 0.713-0.916).

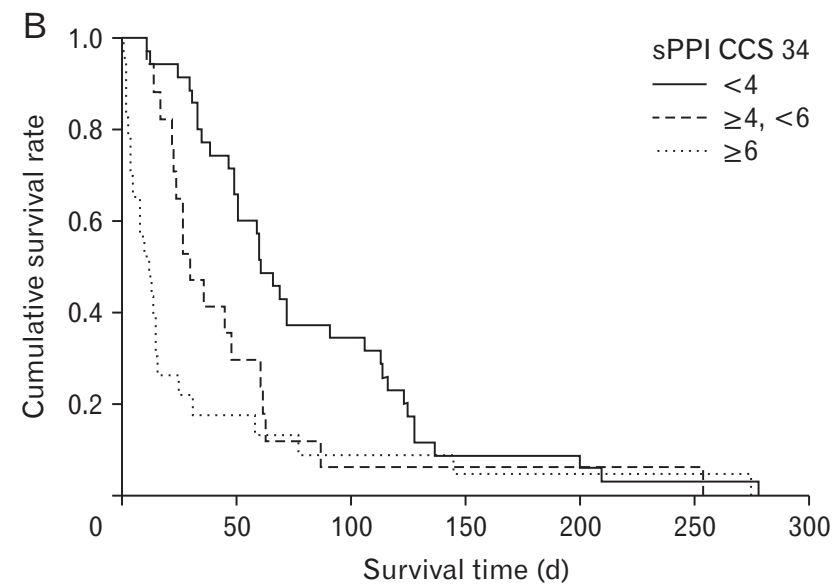

Figure 1. Kaplan-Meier survival curves for each simplified Palliative Prognostic Index (sPPI) group. (A) sPPI1: sPPI including Communication Capacity Scale (CCS) levels 2, 3 , and 4 for the diagnosis of delirium. The median survival time in group A (sPPI $<4, n=24)$, group $B$ (sPPI $\geq 4$ and $<6, n=12$ ), and group $C$ (sPPI1 $\geq 6$, $n=39$ ) was 60 days (95\% confidence interval [Cl], 39.8-80.1), 27 days (95\% Cl, 13.4-40.6), and 16 days (95\% Cl, 3.8-28.2), respectively ( $\mathrm{P}=0.007$ for log-rank test). (B) sPPI2: sPPI including CCS levels 3 and 4 for the diagnosis of delirium. The median survival time in group $A$ (sPPI2 $<4, n=35$ ), group B (sPPI2 $\geq 4$ and $<6, n=17$ ), and group $C$ (sPPI3 $\geq 6, n=23$ ) was 61 days $(95 \% \mathrm{Cl}, 49.4-72.6), 30$ days $(95 \% \mathrm{Cl}, 17.9-42.1)$, and 12 days (95\% $\mathrm{Cl}, 4.2-19.8)$, respectively (P=0.006 for log-rank test). (C) sPPI3: sPPI including the Korean Nursing Delirium Screening Scale (NuDESC) $\geq 2$ points for the diagnosis of delirium. The median survival time in group $A$ (sPPI3 $<4, n=32$ ), group $B$ (sPPI3 $\geq 4$ and $<6, n=16$ ), and group $\mathrm{C}(\mathrm{sPPI} 3 \geq 6, \mathrm{n}=26)$ was 60 days $(95 \% \mathrm{Cl}, 50.3-69.7)$, 27 days $(95 \% \mathrm{Cl}, 11.3-42.7)$, and 16 days $(95 \% \mathrm{Cl}, 7.8-20.6)$, respectively ( $\mathrm{P}=0.002$ for log-rank test). 
Table 3. Accuracy of prediction for 3- and 6-week survival using the sPPI

\begin{tabular}{|c|c|c|c|c|c|}
\hline Predicted survival & Sensitivity & Specificity & PPV & NPV & $\mathrm{OA}$ \\
\hline \multicolumn{6}{|l|}{$\leq 3$ weeks } \\
\hline sPPI $1 \geq 6$ & 0.909 & 0.642 & 0.513 & 0.944 & 0.720 \\
\hline $\mathrm{sPPI} 2 \geq 6$ & 0.773 & 0.887 & 0.739 & 0.904 & 0.854 \\
\hline sPPI3 $\geq 6$ & 0.762 & 0.811 & 0.615 & 0.896 & 0.797 \\
\hline \multicolumn{6}{|l|}{$\leq 6$ weeks } \\
\hline sPPI1 $\geq 4$ & 0.868 & 0.514 & 0.647 & 0.792 & 0.693 \\
\hline sPPI2 $\geq 4$ & 0.763 & 0.703 & 0.725 & 0.743 & 0.734 \\
\hline sPPI3 $\geq 4$ & 0.784 & 0.649 & 0.690 & 0.750 & 0.716 \\
\hline
\end{tabular}

SPPI1: sPPI including CCS levels 2, 3, and 4 for the diagnosis of delirium; sPPI2: sPPI including CCS levels 3 and 4 for the diagnosis of delirium; sPPI3: sPPI including the Korean Nursing Delirium Screening Scale $\geq 2$ points for the diagnosis of delirium.

sPPI, simplified Palliative Prognostic Index; PPV, positive predictive value; NPV, negative predictive value; OA, overall accuracy; CCS, Communication Capacity Scale.

\section{DISCUSSION}

In this study, the actual survival time of the three groups, classified according to sPPI scores for the prediction of life expectancy, showed statistical differences when compared using the Kaplan-Meier method and the log-rank test. Predicting 3- and 6-week survival using the sPPI showed acceptable accuracy in sensitivity and specificity, confirming this instrument's utility in predicting the life expectancy of patients with terminal cancer who had received home-based hospice care. This study consistently showed the validity of the sPPI regardless of the diagnostic methods of delirium used instead of the DSM-IV standard.

According to a study by Hamano et al., ${ }^{18)}$ a life expectancy prediction of less than 3 weeks when the sPPI score was $>6$ showed $72.9 \%$ sensitivity, $67.6 \%$ specificity, $56.2 \%$ PPV, and $81.4 \%$ NPV. Moreover, a life expectancy prediction of less than 6 weeks when the sPPI score was $>4$ showed $80.3 \%$ sensitivity, $61.8 \%$ specificity, $73.6 \%$ PPV, and $70.3 \%$ NPV. ${ }^{18)}$ In this study, most values were similar to, if not higher than, those in previous studies. Furthermore, the prognosis of the three groups, classified by sPPI scores of 4 and 6, showed significant differences when compared based on the Kaplan-Meier method and logrank test. The AUCs of the 3- and 6-week survival predictions were all $>0.7$, confirming discriminative accuracy of sPPI.

It is necessary to consider various indicators such as sensitivity, specificity, PPV, and NPV when determining the accuracy of a diagnostic tool. However, it is practically impossible for tools to have high values on all indicators. There is no clear standard for judgment, but empirically, a tool is deemed useful when the sum of the sensitivity and specificity is $150 \%$ or higher. ${ }^{23)}$ The AUC is used to confirm the overall validity of a tool, where the larger the area, the higher the validity. An AUC between 0.9 and 1.0 indicates that the tool is excellent; between 0.8 and 0.9 , very good; between 0.7 and 0.8 , good; between 0.6 and 0.7 , sufficient; between 0.5 and 0.6 , bad; and $<0.5$, not useful. ${ }^{24)}$ Therefore, the sPPI has utility for prognosis prediction. This study is the first of its kind to prove the utility of the sPPI in patients with terminal cancer in South Korea; however, further studies in various environments are necessary for wider application of the sPPI.

Hospice palliative care in South Korea has been centered on inpatient care since the pilot project for patients with terminal cancer in
2003, while home-based hospice care was introduced after the pilot project in 2016. There are few related studies and it is difficult to examine the current situation owing to late introduction of hospice in South Korea. However, the home may be more comfortable than a hospital for both patients and families. According to a study conducted on patients with cancer and their families in eight hospitals in South Korea, about half of the subjects reported that they would rather die at home than in a hospital. ${ }^{25)}$ Moreover, setting these services within the home is necessary to improve the accessibility of hospice care and the turnover of hospice patients in hospitals. Therefore, more studies on patients in home-based hospice care are needed. The sPPI can be particularly useful for home-based hospice care as it facilitates assessment in an environment with limited testing equipment or personnel.

To date, there has been no study on the sPPI for patients receiving home-based hospice care. According to a study by Hamano et al., ${ }^{19}$ which analyzed the utility of the PPI in patients receiving home-based hospice care, a life expectancy prediction of less than 3 weeks when the PPI score was $\geq 6$ points and a life expectancy prediction of less than 6 weeks when the PPI score was $\geq 4$ points showed a sensitivity of $60.0 \%$ and $70.6 \%$ and specificity of $87.0 \%$ and $71.9 \%$, respectively. The sensitivity was lower as compared to values reported by Morita et al., ${ }^{15)}: 83 \%$ and $79 \%$ for 3 - and 6-week survival predictions, respectively. Among the 3- and 6-week survival predictions in this study, the best were those obtained from sPPI2, that is, including CCS levels 3 and 4 for the diagnosis of delirium. It showed a sensitivity of $77.3 \%$ and $76.3 \%$ and specificity of $88.7 \%$ and $70.3 \%$, respectively; these values were higher as compared to previous studies, except for the specificity of the 6-week survival prediction. Furthermore, a multicenter study by Baba et al. ${ }^{14)}$ that analyzed the accuracy of the PaP, D-PaP, PPI, and PiPS showed an accuracy of $69.3 \%-79.5 \%$ across short-term survival ( $\leq 13$ days or $\leq 21$ days) and long-term survival ( $\geq 30$ days, $\geq 42$ days, or $\geq 56$ days) in patients in home-based hospice care. In this study, the accuracy of survival predictions of 3 weeks or less and 6 weeks or less was $72.0 \%-85.4 \%$ and $69.3 \%-73.4 \%$, respectively. Thus, the sPPI is considered to be as accurate as other tools. In particular, when the sPPI score was $\geq 6$, the accuracy of the 3 -week survival prediction was quite high. A future study that directly compares the sensitivity and specificity of the sPPI and other tools would clearly confirm the effec- 
tiveness of the sPPI.

Home-based hospice teams are composed of mandatory personnel such as doctors, nurses, and social workers with certain qualifications, but it is not practical for doctors to regularly visit patients in their homes. According to a study of 49 patients in home-based hospice care conducted at a single institution in South Korea, the number of home visits by doctors per year was $1.143 \pm 0.957$ (mean \pm standard deviation), with a median of once per year. ${ }^{26)}$ Therefore, the main practitioner visiting homes would be a professional hospice nurse. Thus, it is necessary to verify whether there is a significant difference in predictive accuracy regarding patients' life expectancy between doctors and nurses. The Nu-DESC is a tool developed by Gaudreau et al. ${ }^{22)}$ based on the DSM-IV standard. In a study comparing the evaluation of delirium using the Nu-DESC and the DSM-5, sensitivity was $67 \%$ and specificity $93 \%$ when the presence of delirium was defined as a Nu-DESC score of $\geq 1 .{ }^{27)}$ In a study on the Korean Nu-DESC, which was used in this study, the presence of delirium was defined as a score of $\geq 2$, and sensitivity and specificity were $81 \%$ and $97 \%$, respectively ${ }^{20)}$ In this study, the frequency of delirium, judged based on the Korean $\mathrm{Nu}$ DESC, the CCS $1 / 234$, and the CCS $12 / 34$, showed different results, but the sPPI's life expectancy predictive power using the CCS or the Korean Nu-DESC exhibited adequate accuracy. The significance of such findings is that both the CCS and Korean Nu-DESC were evaluated by hospice nurse specialists. The accuracy of the sPPI evaluated by nurses, rather than doctors, was proven, and it was shown that the sPPI may be more useful for patients receiving home-based hospice care where doctors' visits or diagnoses of delirium are difficult.

In addition, a study on the life expectancy of patients with terminal cancer showed that the accuracy of predictions made by multidisciplinary teams, doctors, and nurses was $57.5 \%, 56.3 \%$, and $55.5 \%$, respectively. The predictions made by nurses were less accurate than those of multidisciplinary teams, but they were not significantly different from those made by doctors. Moreover, the predictions made by patients were significantly lower in accuracy than those of clinicians. ${ }^{28)}$ These studies provide evidence that doctors and nurses show a fairly similar level of accuracy when determining the presence of delirium and the prognosis of patients with terminal cancer. Future studies comparing the CCS evaluated by nurses with the actual frequency of delirium, as well as those comparing the results of the sPPI and the PPI evaluated by both doctors and nurses, would help suggest an appropriate cutoff value for the CCS and confirm nurses' predictive power regarding life expectancy.

There are some limitations to this study. First, it is difficult to generalize the conclusions given the sample size $(n=75)$; thus, large-scale studies are required. Second, the study is limited to patients receiving home-based hospice care in a single institution; hence, it is difficult to apply the results to all patients with terminal cancer. However, this study has significance in that it confirmed the applicability of the sPPI for use with South Korean patients. Since there are insufficient data on the sPPI to compare it to other studies, more research in South Korea as well as abroad is necessary. Third, for the classification of CCS lev- els, the frequency of delirium defined as $1 / 234$ (47.4\%) and 12/34 (4.0\%) were both different from the frequency assessed by the Korean $\mathrm{Nu}$-DESC (16\%), and it was difficult to compare the actual frequency of delirium because its diagnosis was not based on DSM criteria. When the PPI was first developed in 1999, it used the DSM-IV criteria, but the DSM-5, which was released in 2013, ${ }^{29)}$ entailed certain changes in diagnostic criteria for delirium. While the DSM-IV mentioned the disturbance of consciousness, the DSM- 5 removed items related to consciousness level, instead focusing on the disturbance in attention and awareness. In a study that diagnosed patients with delirium using each DSM criterion and compared the mortality rate and length of stay, patients diagnosed with delirium using the DSM-5 and the DSMIV accounted for $20.5 \%$ and $22.5 \%$ of the subjects, respectively. The mortality rate was highest when diagnosed using the DSM-5, while the length of stay was longest when diagnosed using the DSM-IV. ${ }^{30)}$ As different outcomes occur when different diagnostic criteria are used, changes must be made when determining delirium, which is an item of the original PPI. Delirium must be diagnosed based on the revised diagnostic criteria according to the DSM-5 while re-evaluating the original PPI. Furthermore, a study comparing delirium diagnosed with the DSM-5 and the CCS is necessary to increase the reliability of the CCS in diagnosing delirium.

Despite such limitations, this study provides grounds for applying the sPPI, a single prognostic index, to patients with terminal cancer by expanding it to South Korean patients. Moreover, this study confirms that the SPPI has reasonable predictive power and is easy to use for hospice nurse specialists, who may not necessarily be doctors, to measure prognoses, thereby expanding the possibility of its clinical use and providing more appropriate palliative care.

\section{CONFLICT OF INTEREST}

No potential conflict of interest relevant to this article was reported.

\section{ORCID}

Hyeon-Jeong Yang: https://orcid.org/0000-0001-9379-9170

Seok-Joon Yoon: https://orcid.org/0000-0002-6784-8191

Jong-Sung Kim: https://orcid.org/0000-0001-5945-0784

Sung-Soo Kim: https://orcid.org/0000-0001-9417-7728

Jin-Gyu Jung: https://orcid.org/0000-0003-1400-4187

Won Yoon Suh: https://orcid.org/0000-0002-8028-1708

Sami Lee: https://orcid.org/0000-0003-3653-7734

Hyun Gu Kim: https://orcid.org/0000-0002-0931-1142

Yong Woo Lee: https://orcid.org/0000-0003-0836-0759

\section{REFERENCES}

1. Steinhauser KE, Christakis NA, Clipp EC, McNeilly M, Grambow S, Parker J, et al. Preparing for the end of life: preferences of patients, families, physicians, and other care providers. J Pain Symptom Man- 
age 2001;22:727-37.

2. Christakis NA, Iwashyna TJ. Attitude and self-reported practice regarding prognostication in a national sample of internists. Arch Intern Med 1998;158:2389-95.

3. Rose JH, O'Toole EE, Dawson NV, Thomas C, Connors AF Jr, Wenger $\mathrm{N}$, et al. Generalists and oncologists show similar care practices and outcomes for hospitalized late-stage cancer patients. Med Care 2000; 38:1103-18.

4. Lau F, Cloutier-Fisher D, Kuziemsky C, Black F, Downing M, Borycki E, et al. A systematic review of prognostic tools for estimating survival time in palliative care. J Palliat Care 2007;23:93-112.

5. Lau F, Maida V, Downing M, Lesperance M, Karlson N, Kuziemsky C. Use of the Palliative Performance Scale (PPS) for end-of-life prognostication in a palliative medicine consultation service. J Pain Symptom Manage 2009;37:965-72.

6. Glare PA, Eychmueller S, McMahon P. Diagnostic accuracy of the palliative prognostic score in hospitalized patients with advanced cancer. J Clin Oncol 2004;22:4823-8.

7. Scarpi E, Maltoni M, Miceli R, Mariani L, Caraceni A, Amadori D, et al. Survival prediction for terminally ill cancer patients: revision of the palliative prognostic score with incorporation of delirium. Oncologist 2011;16:1793-9.

8. Hyodo I, Morita T, Adachi I, Shima Y, Yoshizawa A, Hiraga K. Development of a predicting tool for survival of terminally ill cancer patients. Jpn J Clin Oncol 2010;40:442-8.

9. Gwilliam B, Keeley V, Todd C, Gittins M, Roberts C, Kelly L, et al. Development of prognosis in palliative care study (PiPS) predictor models to improve prognostication in advanced cancer: prospective cohort study. BMJ 2011;343:d4920.

10. Alshemmari S, Ezzat H, Samir Z, Refaat S, Alsirafy SA. The palliative prognostic index for the prediction of survival and in-hospital mortality of patients with advanced cancer in Kuwait. J Palliat Med 2012;15: 200-4.

11. Subramaniam S, Thorns A, Ridout M, Thirukkumaran T, Osborne TR. Accuracy of prognosis prediction by PPI in hospice inpatients with cancer: a multi-centre prospective study. BMJ Support Palliat Care 2013;3:324-9.

12. Stone CA, Tiernan E, Dooley BA. Prospective validation of the palliative prognostic index in patients with cancer. J Pain Symptom Manage 2008;35:617-22.

13. Cheng WH, Kao CY, Hung YS, Su PJ, Hsieh CH, Chen JS, et al. Validation of a palliative prognostic index to predict life expectancy for terminally ill cancer patients in a hospice consultation setting in Taiwan. Asian Pac J Cancer Prev 2012;13:2861-6.

14. Baba M, Maeda I, Morita T, Inoue S, Ikenaga M, Matsumoto Y, et al. Survival prediction for advanced cancer patients in the real world: a comparison of the Palliative Prognostic Score, Delirium-Palliative Prognostic Score, Palliative Prognostic Index and modified Prognosis in Palliative Care Study predictor model. Eur J Cancer 2015;51: 161829.

15. Morita T, Tsunoda J, Inoue S, Chihara S. The Palliative Prognostic In- dex: a scoring system for survival prediction of terminally ill cancer patients. Support Care Cancer 1999;7:128-33.

16. American Psychiatric Association. Diagnostic and statistical manual of mental disorders, 4th edition (DSM-IV). Washington (DC): American Psychiatric Association; 1994.

17. Morita T, Tsunoda J, Inoue S, Chihara S, Oka K. Communication Capacity Scale and Agitation Distress Scale to measure the severity of delirium in terminally ill cancer patients: a validation study. Palliat Med 2001;15:197-206.

18. Hamano J, Morita T, Ozawa T, Shishido H, Kawahara M, Aoki S, et al. Validation of the simplified palliative prognostic index using a single item from the Communication Capacity Scale. J Pain Symptom Manage 2015;50:542-7.

19. Hamano J, Kizawa Y, Maeno T, Nagaoka H, Shima Y, Maeno T. Prospective clarification of the utility of the palliative prognostic index for patients with advanced cancer in the home care setting. Am J Hosp Palliat Care 2014;31:820-4.

20. Kim KN, Kim CH, Kim KI, Yoo HJ, Park SY, Park YH. Development and validation of the Korean Nursing Delirium Scale. J Korean Acad Nurs 2012;42:414-23.

21. Anderson F, Downing GM, Hill J, Casorso L, Lerch N. Palliative performance scale (PPS): a new tool. J Palliat Care 1996;12:5-11.

22. Gaudreau JD, Gagnon P, Harel F, Tremblay A, Roy MA. Fast, systematic, and continuous delirium assessment in hospitalized patients: the nursing delirium screening scale. J Pain Symptom Manage 2005;29: 368-75.

23. Power M, Fell G, Wright M. Principles for high-quality, high-value testing. Evid Based Med 2013;18:5-10.

24. Simundic AM. Measures of diagnostic accuracy: basic definitions. EJIFCC 2009;19:203-11.

25. Choi KS, Chae YM, Lee CG, Kim SY, Lee SW, Heo DS, et al. Factors influencing preferences for place of terminal care and of death among cancer patients and their families in Korea. Support Care Cancer 2005; 13:565-72.

26. Youn KH, Gwak JI, Bang JY, Lee EJ. Change in hospital using pattern after palliative home care program. Korean J Fam Pract 2018;8:386-90.

27. Hargrave A, Bastiaens J, Bourgeois JA, Neuhaus J, Josephson SA, Chinn $\mathrm{J}$, et al. Validation of a nurse-based delirium-screening tool for hospitalized patients. Psychosomatics 2017;58:594-603.

28. Gwilliam B, Keeley V, Todd C, Roberts C, Gittins M, Kelly L, et al. Prognosticating in patients with advanced cancer: observational study comparing the accuracy of clinicians' and patients' estimates of survival. Ann Oncol 2013;24:482-8.

29. American Psychiatric Association. Diagnostic and statistical manual of mental disorders, 5th edition (DSM-5). Washington (DC): American Psychiatric Association; 2013.

30. Adamis D, Meagher D, Rooney S, Mulligan O, McCarthy G. A comparison of outcomes according to different diagnostic systems for delirium (DSM-5, DSM-IV, CAM, and DRS-R98). Int Psychogeriatr 2018;30:5916. 